\title{
Hat die Globalisierung den Siegeszug des Nationalstaats beendet?
}

\section{Einleitung}

Die Humanwissenschaften stecken offensichtlich voller Leute, die begeistert verkünden, eine neue Form der menschlichen Gesellschaft sei im Entstehen begriffen. Die enthusiastischsten Vertreter dieser Spezies vergleichen unsere Gegenwart mit dem 18. Jahrhundert, als Industrielle Revolution, »Modernismus« und »Aufklärung《 angeblich die Gesellschaft revolutionierten. Heute, so ist zu hören, erleben wir die ersten schmerzhaften Ansätze eines vergleichbaren Wandels hin zur »postindustriellen« oder »postmodernen « Gesellschaft. Andere Terminologien lassen an weniger revolutionären Wandel denken. Begriffe wie »Spätkapitalismus«, »Spätmoderne« oder »radikale Moderne « sollen unterschiedliche Grade kontiniuierlichen und weniger abrupten Wandels bezeichnen. Auch neue »Global《-Begriffe werden in die Debatte geworfen: »Globaler Kapitalismus « mag nur die starke Ausweitung einer alten Wirtschaftsform bezeichnen, während »Weltgesellschaft« zumeist ein radikal neues Phänomen in der Geschichte der menschlichen Gesellschaft suggeriert.

$\mathrm{Zu}$ den Enthusiasten zählen ganz unterschiedliche Gruppen von Philosophen, Historikern, Soziologen, Ökonomen, Geographen und Ökologen. Sie stimmen in sehr wenigem überein - vor allem nicht in der Frage, ob sie die Veränderungen gutheißen sollten. Doch in einem Punkt sind sie sich einig: die neuen Entwicklungen schwächen den Nationalstaat. Von Theoretikern der Postmoderne wie Baudrillard, Lyotard und Jameson über Geographen wie Harvey und Taylor, Soziologen wie Giddens, Lash und Urry bis hin zu den Ökonomen, die im Economist ihr Sprachrohr haben - von allen Seiten wird verkündet, der Nationalstaat werde »untergraben «, »unterlaufen «, »umgangen « oder »marginalisiert« (neuere Beispiele sind Taylor 1996, Lash/ Urry 1994, Featherstone 1990, Harvey 1990, The Economist 1995). Nur in einer Hinsicht wird diese These manchmal eingeschränkt. Weil das »Ethnische« in den Szenarien der »postmodernen Zersplitterung« eine mächtige 
Rolle spielt, wird oft ein Wiederaufleben des Nationalismus verkündet. Doch für den alten Nationalstaat finden sich zumeist nur noch Nachrufe. Viele der Enthusiasten sind Westeuropäer - was nicht überrascht, da gerade diese Region des Globus die meiste politische Unterstützung für ihre Grabreden auf den Staat liefert. Viele von ihnen (Marxisten wie Neoklassiker) sind Materialisten, die auf die sich anbahnenden großen Veränderungen im Kapitalismus verweisen und glauben, diese würden unweigerlich auch den Rest der Gesellschaftsstruktur umkrempeln. Die meisten Argumente beruhen im Kern auf den technischen und informationellen Neuerungen der Gegenwart. Transport- und Informationssysteme, die schnellen (oft sofortigen) $\mathrm{Zu}$ gang zur Welt bieten, stellen die Infrastrukuren einer Weltgesellschaft dar. Ich bestreite nicht, daß diese potentielle Infrastruktur einer Weltgesellschaft schon existiert: die Logistik der Kommunikation und von daher der Macht befindet sich tatsächlich in einem revolutionären Wandel. Personen, Waren und besonders Informationen zirkulieren rund um den Globus, und von daher ist die von den Enthusiasten propagierte Vision einer Weltgesellschaft technisch durchaus möglich. Doch ist sie auch schon Wirklichkeit? Um dies zu belegen, stellen diverse Gruppen von Enthusiasten vier Kernthesen auf: (1) Der Kapitalismus, inzwischen global, transnational, postindustriell, »informationell«, konsumistisch, neoliberal und »restrukturiert«, untergräbt den Nationalstaat - die makroökonomische Planung, den kollektivistischen Sozialstaat, die Vorstellungen der Bürger von einer kollektiven Identität, überhaupt die Einhegung des sozialen Lebens durch den Staat.

(2) Andere Aspekte der »Globalisierung «, besonders die aus der Umweltverschmutzung und dem Bevölkerungswachstum entstehenden Gefährdungen, die womöglich zu einer neuen »Risikogesellschaft« führen, sind zu allgemein und zu schwerwiegend geworden, als daß der Nationalstaat alleine mit ihnen fertig werden könnte.

(3) Die »Identitätspolitik « und die »neuen sozialen Bewegungen«, die neue Technologien einsetzen, verschärfen das Profil diverser lokaler und transnationaler Identitäten auf Kosten nationaler Identitäten wie auch jener breiten Klassenidentitäten, mit denen es der Nationalstaat traditionell zu tun hatte. Aus diesem und dem zuvor genannten Grund erleben wir die ersten Regungen einer neuen, transnationalen "Zivilgesellschaft«, sozialer Bewegungen für Frieden, Menschenrechte und ökologische und soziale Reformen in wirklich globaler Dimension.

(4) Durch das Ende der nuklearen Konfrontation der Weltmächte wird die staatliche Souveränität und die »harte Geopolitik« untergraben. Zwar verlief die Expansion des modernen Nationalstaats größtenteils über Kriege, für die die ganze Bevölkerung mobilisiert wurde, doch gerade dies ist heute irrational. Martin Shaws Konzept der Entwicklung eines »Weltstaates« ist 
die vielleicht ausgewogenste Version dieser These (Shaw 1996). Allerdings handelt es sich um eine dezidierte Minderheitsposition auf dem akademischen Feld der Internationalen Beziehungen, wo man zumeist noch dem Studium des souveränen Staats verhaftet ist.

Der empirische Teil des vorliegenden Artikels wird sich mit diesen vier Thesen zur Schwächung des Nationalstaats auseinandersetzen. Da ihre Vertreter die politischen Machtverhältnisse herunterspielen, werden im folgenden auch zwei politiktheoretische Gegenthesen erörtert.

(A) Staatliche Institutionen, seien sie nun innen- oder geopolitisch ausgerichtet, besitzen immer noch kausale Wirksamkeit, da sie (wie auch ökonomische, ideologische und militärische Institutionen) die notwendigen Bedingungen für die gesellschaftliche Existenz bereitstellen': die Regulierung von Aspekten des gesellschaftlichen Lebens, die eindeutig 》territorial eingegrenzt« sind (Mann 1986, Kap. 1). Von daher können sie nicht die bloße Folge anderer Quellen gesellschaftlicher Macht sein.

(B) Da die Staaten recht heterogen sind, werden, falls (A) wahr ist, die vorhandenen Unterschiede auch verschiedene Folgen in anderen gesellschaftlichen Sphären zeitigen. Selbst in Europa unterscheiden sich die Staaten nach Größe, Macht, Geographie und Zentralisationsgrad. Rund um den Globus finden sich noch dramatischere Unterschiede: Grad der Demokratisierung, Entwicklungsniveau, infrastrukturelle und geopolitische Macht, nationale Verschuldung usw. In den einzelnen Ländern finden sich außerdem ganz unterschiedliche regionale Verhältnisse. Kann der gegenwärtige Kapitalismus, selbst wenn er durch Umweltgefahren, die »kulturelle Postmoderne« und die Entmilitarisierung verstärkt wird, all diese Unterschiede neutralisieren und auf alle Länder dieselben Wirkungen ausüben? Oder werden die Unterschiede zu Unterschieden in diesen Wirkkräften führen und damit die Globalisierung begrenzen?

Nur die atemlosesten Enthusiasten würden diesen Gegenthesen jegliche Triftigkeit absprechen - oder dem Überleben des Nationalstaats als Verfügungsgewalt über gewisse ökonomische, ideologische, militärische und politische Ressourcen. Es geht darum, Grade relativer Kausalität auszumachen: In welchem Maße wird der Nationalstaat umgestaltet, in welchem Maße verliert er seine Rolle - oder wächst sie etwa immer noch?

Um diese Fragen zu beantworten müssen wir auch einige begriffliche Unterscheidungen treffen. Grob können wir fünf sozio-räumliche Netze gesellschaftlicher Interaktion in der heutigen Welt unterscheiden.

1 Natürlich gab es auch staatenlose Gesellschaften (die das menschliche Leben über lange Zeiten hinweg prägten), und es gibt sie auch heute noch. Doch für fortgeschrittenes gesellschaftliches Leben sind offenbar Staaten nötig - auch wenn die Anarchisten hier nicht zustimmen werden. 
(1) Lokale Netzwerke - für unsere Zwecke sind dies einfach subnationale Interaktionsnetzwerke.

(2) Nationale Netzwerke, die durch den Nationalstaat strukturiert oder (neutraler formuliert) eingegrenzt werden.

(3) Internationale Netzwerke, d.h. Beziehungen zwischen national konstituierten Netzwerken. Auf den ersten Blick gehört dazu die »nationale Geopolitik « der zwischenstaatlichen Beziehungen, die sich um Krieg, Frieden und Bündnisse dreht. Doch auch die »weiche Geopolitik« zwischen den Staaten gehört dazu: Verhandlungen über weniger konfliktträchtige und eher partikulare Angelegenheiten wie die Flugverkehrsstrecken, Steuerabkommen, Luftverschmutzung usw. Desgleichen gehören dazu Beziehungen zwischen Netzwerken, die eher national als staatlich konstituiert sind: die »nationalen Champions« etwa, die sich zunehmend auf größeren Spielfeldern betätigen - seien es nun Fußballteams oder Großunternehmen.

(4) Transnationale Netzwerke überschneiden nationale Grenzen, die für sie ohne Belang sind. Sie mögen nicht sehr ausgedehnt sein - etwa eine religiöse Sekte, die sich auf dem Gebiet zweier Länder organisiert hat - oder auch einen ganzen Kontinent, gar die Welt umspannen. Viele Argumente aus der transnationalen Ecke beziehen sich auf »makroregionale « Verhältnisse, so etwa die Unterscheidung von »liberal/angelsächsischen «, »nordisch/sozialdemokratischen « und »christdemokratisch/korporatistischen« Formen des Sozialstaats.

(5) Globale Netzwerke überspannen die ganze Welt - oder, vielleicht etwas wirklichkeitsnäher, den größten Teil des Globus. Doch sollten wir zwischen Netzwerken unterscheiden, die sich universalistisch, und solchen, die sich partikularistisch über den Globus erstrecken. Die feministische Bewegung mag sich in fast allen Ländern verbreiten, doch meist nur unter partikularen, kleineren Gruppen. Die katholische Kirche ist auf allen Kontinenten mehr oder weniger präsent, in Asien besitzt sie jedoch nur eine schmale Basis, während sie in Lateinamerika fast unausweichlich ist. Der Kapitalismus, an den die Enthusiasten gerne erinnern, ist ein universelles globales Netzwerk, der fast überall gleichmäßig das wirtschaftliche und gesellschaftliche Leben durchdringt. Globale Netzwerke können also entweder durch ein einziges universelles Netzwerk gebildet werden oder durch eine stärker segmentierte Reihe von Netzwerken, zwischen denen eher partikularistische Beziehungen bestehen.

Während der letzten Jahrhunderte hat das relative Gewicht der lokalen Netzwerke eindeutig abgenommen; zugleich haben die über größere Distanzen sich erstreckenden Netzwerke - nationale, internationale und transnationale - an Dichte gewonnen und strukturieren heute in höherem Maße das Leben der Menschen. Wirklich globale Netzwerke entstehen erst seit 
relativ kurzer Zeit. Festzustellen ist noch einmal, daß globale Netzwerke nicht zugleich auch transnationale Netzwerke sein müssen, auch wenn viele Enthusiasten sie gleichsetzen. Auch haben sie nicht unbedingt ökonomischen Charakter. Globale Netzwerke können (wie Shaw ausführt) durch Geopolitik konstitutiert werden oder durch ideologische Bewegungen, die religiös, sozialistisch, feministisch oder neoliberal inspiriert sind - deren Kombination womöglich eine neue transnationale Zivilgesellschaft ergibt.

Da nationale und internationale Netzwerke durch den Nationalstaat konstituiert oder auf fundamentale Weise eingegrenzt werden, hängt die Zukunft des Nationalstaat entscheidend von den Antworten auf zwei Fragen ab: Schwindet die gesellschaftliche Bedeutung der nationalen und internationalen Netzwerke im Vergleich zu lokalen und transnationalen Netzwerken? Und welchen Beitrag leisten die nationalen/internationalen bzw. die lokalen/transnationalen Netzwerke zur Entwicklung globaler Netzwerke?

\section{Der »bescheidene Nationalstaat « des Nordens}

Die bekannteste und vorherrschende Form des Staates in der heutigen Welt entstand im »Westen«, genauer im »Nordwesten« Westeuropas und seiner weißen Kolonien: ein Staat, der formelle politische Souveränität über »seine« Gebiete und eine Legitimität beanspruchte, die im jeweiligen »Volk« oder in der »Nation« gründete. Dies meinen wir mit Nationalstaat. Im Laufe mehrerer Jahrhunderte wuchs die Regulationsmacht solcher Staaten. Seit dem Ende des Mittelalters beanspruchten sie mit zunehmender Plausibilität das Monopol juristischer Regulation und militärischer Gewalt. Später, im 18. und vor allem im 19. Jahrhundert, finanzierten sie die Verflechtung der Verkehrs- und Kommunikationsinfrastruktur und die elementare Kontrolle der Armen. Im 20. Jahrhundert entwickelten sich Wohlfahrtsstaaten, makroökonomische Planung und die Massenmobilisierung nationaler Bevölkerungen. Unterdessen legitimierten sich immer mehr Staaten unter Berufung auf »das Volk«, entweder »repräsentativ« (liberale Demokratien) oder als seine »organische Verkörperung « (autoritäre Regime), bei unterschiedlichen Ausprägungen ziviler, politischer und sozialer Staatsbürgerschaft. Die Bevölkerungen des Nordwestens wurden damit in gewissem Maße in nationale Interaktionsnetzwerke »eingeschlossen«, die dann durch die als »Geopolitik« bekannten internationalen Beziehungen von Nationalstaaten ergänzt wurden.

Dies ist die heute bekannte Geschichte vom $»$ Siegeszug « des Nationalstaats und des Systems der Nationalstaaten - zu dem ich mich an anderer Stelle geäußert habe (Mann 1986, 1993a). Doch sollten wir festhalten, daß die Expansion dieser nationalen und internationalen Netzwerke immer einherging mit der Expansion bestimmter »transnationaler« Machtbeziehungen, 
besonders jener des Industriekapitalismus und seiner Begleitideologien (Liberalismus, Sozialismus), sowie der breiteren kulturellen Netzwerke, die im Nordwesten von europäisch/christlichen »weißen« Vorstellungen kollektiver Identität gebildet wurden. Daher wuchsen nationale und internationale Interaktionsnetzwerke stärker auf Kosten der lokalen als der transnationalen Netzwerke. In derselben Periode des späten 19. Jahrhunderts etwa, als die europäischen Staaten ihr nationales Bildungssystem und das öffentliche Gesundheitswesen ausbauten, Zölle anhoben und sich den Kriegen näherten (Beispiele nationaler und internationaler Einschließung), boomte der transnationale Handel und erreichte schließlich einen Anteil an der globalen Produktion, der dem heutigen gleichkommt. Getragen von einem blasierten kulturellen Überlegenheitsgefühl arbeiteten die Mächte des Nordwestens auch zusammen, um die Chinesen zu christianisieren, auszubeuten und mit Drogen zu beliefern. Im Grunde setzte das Wachstum der Nationalstaaten eine weitere globale Expansion voraus, vorderhand um es zu finanzieren, aber vielleicht auch, weil eine Vorstellung von Nation die Vorstellung einer europäisch-christlich-weißen Überlegenheit vorausgesetzt haben mag, die alle Klassen des Nordwestens und beide Geschlechter mit einem Gefühl des moralischen Werts und der Gleichheit ausgestattet hat. Der letzte große expansionistische Schub des Nationalstaats, von 1945 bis in die sechziger Jahre, könnte durchaus beide Elemente benötigt haben. Die Staaten hatten dank der gewaltigen ökonomischen Expansion üppige Mittel und besaßen auch die im Krieg und in der Aufbauphase entstandenen Institutionen, um diese Mittel ausgeben zu können. Da die nordwestlichen Nationen ihren Kolonien die Werte der »Zivilisation« beigebracht hatten, "gewährten« sie ihnen nun die Unabhängigkeit in ihrer europäischen Gestalt, dem Nationalstaat. In der Vergangenheit entwickelten sich der transnationale Kapitalismus und die kulturellen Identitäten zusammen mit dem Nationalstaat und seinem internationalen System. Auch herrschte ein komplexes Verhältnis von relativer Autonomie und symbiotischer Interdependenz.

Die meisten Staaten des Nordwestens verloren während ihrer langen Expansionsphase auch gewisse Funktionen. Im Laufe ihrer »Säkularisierung« gaben sie Befugnisse moralischer Regulation ab, die sie im Prinzip gemeinsam mit den Kirchen besessen hatten (obwohl die moralische Regulation früherer Jahrhunderte durch kirchliche und nicht staatliche Instanzen umgesetzt wurde). Von Bedeutung ist ebenfalls, daß ein Großteil des Wirtschaftslebens nie in den Machtbereich des Staates geraten war: der Titel dafür ist »Privat«-Eigentum. Weite Teile des gesellschaftlichen Lebens blieben privat oder wurden es zusehends, außerhalb des nationalstaatlichen Kompetenzbereichs, selbst während seiner großen Expansionsphase. Das Eigentum blieb privat, die Schwulen blieben hinter verschlossener Tür. Ka- 
pitalismus und Moral waren im Grunde autonom gegenüber dem Staat. Weiter unten werde ich die These aufstellen, daß die moralische Autonomie gegenwärtig abnimmt.

Im Nordwesten gelangte also nur ein »gemäßigter Nationalstaat« zur Vorherrschaft. Im Lauf des 20. Jahrhundert besiegte er drei Rivalen. Der eine war das »multinationale Reich«: die Dynastien der Habsburger, der Romanows und der Osmanen, mit schwächeren Staaten und nationalen Identitäten - eine weniger »nationalstaatliche« Alternative. Doch die anderen beiden besiegten Staatsmodelle waren viel stärker national orientiert. Der Faschismus strebte ein mächtigeres, autoritäres Staatsgebilde an, welches das vermeintliche Wesen einer strenger ethnisch abgegrenzten Nation verkörpern sollte. 1945 war der Faschismus diskreditiert - zumindest für die beiden folgenden Generationen. Auch der Staatssozialismus bemühte sich um einen stärkeren Staat (der eine Übergangsphase darstellen sollte). Obwohl er nicht streng nationalistisch geprägt war, nahm er in seiner wachsenden Neigung, das Proletariat mit einem umfassenderen »Volk« oder den »Massen« gleichzusetzen, ein ähnliches Legitimationsprinzip in Anspruch. Ökonomische Autarkie und rigorose Überwachung verstärkten hier noch die »nationale« Einschließung. Es dauerte länger, bis er diskreditiert war, doch dieser Prozeß scheint 1991 (zumindest vorläufig) zum Abschluß gekommen zu sein. Beide Spielarten besiegter Regime beanspruchten - im Gegensatz zu den »gemäßigten Nationalstaaten « - auch ein moralisches Monopol. In der Verantwortung des Staates lag es, den »Sowjetmenschen« heranzuziehen oder den »deutschen Volksgenossen«. Hätten diese ambitionierteren Modelle des »Nationalstaats « den Sieg davongetragen und wäre der Globalisierungsprozeß dennoch in Gang gekommen, würde die Weltgesellschaft aus zwei getrennten Reihen globaler Netzwerke bestehen, zwischen denen stark partikularistische und vermutlich kriegsähnliche Zustände herrschen würden. Da dieser Fall nicht eingetreten ist, steht zu erwarten, $\mathrm{da} ß$ die weitere Globalisierung eher universalistischen Charakter haben wird. Seit 1945 breitete sich der gemäßigte Sieger auf fast dem ganzen restlichen »Norden« aus, d.h. auf dem gesamten europäischen Kontinent und auf wachsenden Bereichen des Ostens und Südasiens. Seine offiziellen Gütesiegel beherrschen auch »den Süden«, während sich alle Staaten in einem Forum namens »Vereinte Nationen « versammeln. Auf den ersten Blick beherrscht der gemäßigte Nationalstaat den ganzen Erdball. In begrenztem Maße trifft dies auch zu. Nur wenige Staaten gründen ihre Legitimität nicht auf die Nation oder haben kein Monopol der inneren Gewalt oder reale territoriale Grenzen. Fast allen gelingt es, politische Maßnahmen zur Bevölkerungskontrolle durchzusetzen und das Gesundheits- und Bildungswesen zu regulieren. Sinkende Sterblichkeit und zunehmende Alphabetisierung mö- 
gen viele Ursachen haben, doch einige sind im Bereich wirksamer staatlicher Politik zu suchen. Aus diesen Gründen werde ich im folgenden die heutigen Staaten als Nationalstaaten bezeichnen. Doch die meisten davon haben eher begrenzte Kontrollmacht über ihre Gebiete und Grenzen, während ihre Ansprüche, die jeweilige Nation zu repräsentieren, oft trügerisch sind. Für weite Teile der Welt bleibt ein echter Nationalstaat mehr Ziel als Realität. Der Aufstieg des Nationalstaats war zwar global, nahm jedoch sehr unterschiedliche Züge an. Der gemäßigte Nationalstaat beherrscht inzwischen den »Norden«, dessen Länder ihm zum Teil auch ihre Expansion verdanken, und stellt für die meisten Völker der Welt ein ersehntes Ziel dar. Ist all dies nun in Gefahr?

\section{Die kapitalistische Bedrohung}

Die Enthusiasten stellen zurecht fest, daß der Kapitalismus einige wichtige Transformationen durchläuft. Hier muß nicht näher erläutert werden, daß er neue »informationelle« und »postindustrielle« Technologien einsetzt, um sich fast über den gesamten Globus auszudehnen und das gesellschaftliche Leben noch tiefer zu durchdringen. Doch wie groß ist die Gefahr, die er für den Nationalstaat darstellt? Und wie "global« und/oder »transnational« ist er eigentlich?

In einem formellen geographischen Sinn ist der Kapitalismus heute tatsächlich mehr oder weniger global. Zwei große geopolitische Ereignisse haben diese massive Expansion ermöglicht. Erstens wurde durch die Entkolonialisierung die Aufteilung der Weltwirtschaft in verschiedene imperiale Zonen beendet. Zweitens hat der Kollaps der sowjetischen Autarkie die Möglichkeit eröffnet, weite Teile Eurasiens kapitalistisch zu durchdringen. Nur der Iran, China und eine Handvoll kleiner kommunistischer Länder erhalten gegenwärtig Teilblockaden aufrecht, von denen man aber annehmen kann, daß sie bald zusammenbrechen werden. China behält im Augenblick besondere Eigentumsformen bei (eine Mischung aus privatem mit verschiedenen Formen staatlichen Eigentums und entsprechender Kontrolle), und es gibt immer noch (im Niedergang begriffene) vereinzelte, über den Globus verteilte Gebiete der Subsistenzwirtschaft. Doch der kapitalistische Warentausch hat eindeutig die Vorherrschaft. Ohne einen selbstbewußten Gegner in Sicht wird der Kapitalismus - wenn auch in eingeschränktem Sinne - global. Dies war weder 1940 noch 1980 der Fall. Offensichtlich handelt es sich um eine grundlegende Transformation.

Aber sind die globalen Netzwerke des Kapitalismus »rein« in dem Sinne, daß sie allein universellen Charakter haben, oder tragen auch andere, eher partikularistische gesellschaftliche Organisationsprinzipien zu ihrer Konstitution bei? Eine Ökonomie mag global sein, doch diese Eigenschaft kann 
ihr vermittels nationaler und internationaler Interaktionsnetzwerke zuwachsen. Schließlich sind mehr als $80 \%$ der Weltproduktion immer noch für die Binnenmärkte bestimmt. Da die Statistiken sich auf den Nationalstaat beziehen, ist nicht bekannt, welchen Beitrag dazu die in eigentlichem Sinne nationalen Tauschprozesse im Vergleich zu dem der verschiedenen lokalen Netzwerke leisten. Die nationale Ökonomie ist vermutlich weniger integriert als die Statistik vermuten läßt - besonders in den unterentwickelten Ländern und in bestimmten Regionen größerer fortgeschrittener Länder wie den USA und Australien. Doch der Nationalstaat strukturiert auf systematische Weise viele ökonomische Netzwerke. Eigentum, Anlagen, Forschung \& Entwicklung vieler »multinationaler « Unternehmen (etwa Banken, Pensionsfonds und Versicherungsfirmen) bleiben überwiegend im »Heimatstaat«, den sie für Humankapital (Erziehung und Bildung), Verkehrs- und Kommunikations-Infrastrukturen und wirtschaftlichen Protektionismus in Anspruch nehmen (Carnoy 1993; Castells 1993). Doch selbst bei den stärker national verankerten Multis sind Vertriebsbereich, Produktionsorganisation und Investitionsströme in hohem Maße transnational. Strategische Allianzen mit Unternehmen anderer »Nationalität« schießen nun aus dem Boden und schwächen die nationale Identität des Eigentums - obwohl viele dieser Arrangements eingegangen werden, um dem Protektionismus zu entgehen und mit dessen Niedergang ebenfalls zerfallen würden.

Die Finanzströme haben weit eher transnationalen Charakter, wie sich anhand der wachsenden Komplexität der Finanzmärkte und der Modelle zeigt, die sie erklären sollen - von den Zufallsmechanismen des »Random Walk « bis zur Chaostheorie! Doch den entsprechenden Institutionen eignet weiterhin eine bürokratische Regelhaftigkeit, in weiten Teilen mit deutlich nationalen Zügen. Die Angestellten von Nikko Europe beginnen ihren Arbeitstag bevor die Tokioter Börse schließt. Sie geben die neuesten Informationen zunächst an ihre europäischen Kunden weiter, die in Wahrheit zumeist japanische Firmen sind. Dann, sobald Wall Street erwacht, werden die Informationen nach Westen geschickt, und London schließt für die Nacht. Auch die Finanzmärkte weisen eine Dualität von nationalem und transnationalem Charakter auf. Einerseits ist der Handel in Staatsanleihen, Währungen, Futures und der Großhandel zwischen den Banken weitgehend transnational und wird oft »offshore « abgewickelt, um die Staatsgrenzen und Kontrollen zu umgehen. Andererseits sind Unternehmensaktien meist auf bestimmte nationale Aktienmärkte begrenzt und unterliegen dem nationalem Unternehmensrecht und verschiedenen Bilanzierungsverfahren (Wade 1996).

Natürlich ist Westeuropa transnationaler geworden; der Kontinent leistet ökonomische Integration in einzigartigem Ausmaß. Hier findet sich ein 
echter einheitlicher Markt, ein Trend, der während der nächsten zwanzig Jahre (zumindest im Kern) vermutlich in eine Einheitswährung münden wird sowie in vorwiegend »europäische« und weniger in national orientierte protektionistische Aktivitäten. Die »national führenden« Unternehmen werden zu »europaweit« führenden, wobei ihnen die EU-Administration wie auch der Nationalstaat unter die Arme greifen wird.

Offensichtlich sollten komplexe ökonomische Fragen dieser Art eingehender untersucht werden, als ich es hier tun kann. Doch zwei Punkte werden deutlich: Europa ist ein Extremfall (den ich später eingehender erörtern werde), und die realen kapitalistischen Interaktionsnetzwerke bleiben im Kern bei der Symbiose von Nationalem und Transnationalem.

Auch ein dritter Umstand wird deutlich: Die meisten »transnationalen« Wirtschaftsbeziehungen können nicht umstandslos mit einem globalen Universalismus gleichgesetzt werden. Die kapitalistische Wirtschaftstätigkeit ist vorwiegend »trilateral« und nicht global, da sie in den drei Regionen des fortgeschrittenen »Nordens«, Europa, Nordamerika und Ostasien konzentriert ist. Hier spielen sich über $85 \%$ des Welthandels ab, über $90 \%$ der Produktion in den hochentwickelten Sektoren wie Elektronik, und hier sitzen, mit Ausnahme einer Handvoll, die 100 größten Multis (Banken eingeschlossen). Das heißt nicht unbedingt, der Kapitalismus sei nicht global. Vielleicht wird hier nur deutlich, daß der Norden reich und der Süden arm ist - und daß beide $\mathrm{zu}$ einem globalen Interaktionsnetzwerk zusammengeschlossen sind. Daraus folgern läßt sich allerdings, daß der Kapitalismus eine geo-ökonomische Ordnung beibehält, die von den Ökonomien der fortgeschrittenen Nationalstaaten beherrscht wird. Die stratifikatorische Ordnung des Kapitalismus wird durch Gruppierungen von Nationalstaaten getragen. Unter anderem schützt dies die Bürger des Nordens: Das schlecht ausgebildete Kind eines ungelernten Arbeiters in Großbritannien oder den USA hat in der Regel bessere materielle Lebensbedingungen (und zwanzig Lebensjahre mehr) als sein Altersgenosse in Brasilien oder Indien. Gewiß verschärfen sich die Ungleichheiten in all diesen Nationalstaaten, doch daß der Großteil der Privilegien der Staatsbürger des Nordens wegfallen könnte, ist fast undenkbar. Dies würde zu einem $\mathrm{Ma}$ gesellschaftlicher Unruhe führen, das mit einem stabilen und profitablen Kapitalismus nicht zu vereinbaren ist. Der Nationalstaat trägt einen Teil der Struktur und der Stratifikation der globalen Netzwerke des Kapitalismus. Zwar herrscht die Ware, aber - besonders im Norden - nur in Verbindung mit der Herrschaft der Staatsbürgerschaft.

Die globale Ökonomie ist auch Gegenstand von lockeren und überwiegend »weichen« internationalen Regulationsbemühungen etwa der G 7, des GATT, der Weltbank oder des IWF. Der Norden beherrscht auch diese Institutionen. Einige beschäftigen sich mit offenbar endlosen Verhandlungen zur Li- 
beralisierung des Welthandels - die vermutlich noch lange dauern werden, da die nationalen Regierungen in jüngster Zeit neben Zöllen noch weitere Barrieren errichtet haben. Wir können noch lange nicht von globalem Freihandel reden, doch vielleicht nähern wir uns diesem gegenwärtig ideologisch vorrangigen Ziel. Aber handelt es sich hier nicht um eine weitere Liberalisierungsphase in der normalen historischen Oszillation um den mittleren Bereich zwischen Freihandel und Protektionismus? Das hängt vom Ausgang anderer Entwicklungen ab, die in diesem Papier erörtert werden. Im Moment also und vermutlich auch in nächster Zukunft gewinnt eine sich rapide globalisierende Ökonomie ihre Eigenschaften nicht nur aus transnationalen Netzwerken. Was das Globale letztlich ausmacht ist ein sehr komplexer Mix des Lokalen und des Nationalen, des Internationalen (in meiner Diskussion vor allem durch den Trilateralismus des Nordens repräsentiert) - und des eigentlich Transnationalen. Die transnationale Ware beherrscht den Globus nicht.

Mit der Zeit mögen manche dieser nationalen und internationalen Strukturierungen schwächer werden. Der herrschaftliche Griff des Nordens auf die Weltwirtschaft könnte sich aufgrund des Drucks komparativer Vorteile lockern. Abgesehen vom High-Tech-Bereich könnten viele produktive Unternehmen ihre Aktivitäten in den kostengünstigeren Süden verlagern und damit zu einer weiteren Globalisierung beitragen (allerdings ohne unbedingt die soziale Ungleichheit zu abzumildern). Doch bisher folgen die Verschiebungen nicht einer (vielleicht zufallsbestimmten) »transnationalen« Logik, sondern einer Kombination von vier Prinzipien: Verfügung über natürliche Ressourcen, geographische Nähe (benachbarte Länder), geopolitische Allianzen (befreundete Länder) und staatliche und zivilgesellschaftliche Stabilität (berechenbare Länder). Während der erste Faktor recht zufällig über den Globus verteilt ist - so kann man alleine mit Öl ziemlich rückständige und entlegene Länder entwickeln - hängen die letzten drei Faktoren meist miteinander zusammen. Die historische Entwicklung der bedeutenden Ökonomien des Nordens verlief im Kontext regionaler Bedingungen, von denen benachbarte Staaten und Gesellschaften ebenfalls profitierten. Die Expansion ging also eher in Richtung der Koreas und Mexikos - freundlich gesinnte Nachbarn mit relativ entwickelten Nationen und Staaten - als etwa in Richtung der meisten afrikanischen Länder. Auch das Wachstum nimmt zumeist keine regionale, »enklavenförmige« Gestalt innerhalb von Staaten an (außer wenn Rohstoffe wichtig sind oder die Ausdehnung über eine Grenze erfolgt und die benachbarte Regierung »Wirtschaftsregionen« subventioniert). Die Entwicklung diffundiert meist über die Kernterritorien dieser Staaten hinaus und unterstützt auch die Entwicklung ihrer Zivilgesellschaften und ihre Tendenz zum Nationalstaat. 
Diese Ausdehnung des Nordens - und ebenfalls die Globalisierung - hängt davon $a b$, daß die Nationalstaaten davon profitierten. Diese Form der Globalisierung stärkt also die Nationalstaaten und die nationalen Netzwerke.

Da das Finanzkapital offenbar transnationaler ist als das Industriekapital, werden die Zwänge, die es dem Nationalstaat auferlegt, von den Enthusiasten zumeist am deutlichsten betont. Seine Mobilität und Geschwindigkeit führt zu Finanzbewegungen, welche die fiskalischen Ressourcen der Staaten winzig aussehen lassen und zwei der drei Mechanismen der Fiskalpolitik der Nachkriegszeit in die Schranken weisen - die Zins- und die Währungspolitik (weniger die Besteuerung). Allerdings ist es aus zwei Gründen schwierig, die Bedeutung dieses Phänomens insgesamt abzuschätzen. Erstens bieten die Zahlen keine genaue Auskunft über die Kräfteverhältnisse. Da Währungen, Aktien, Futures usw. an einem einzigen Tag viele Male gehandelt werden können, übersteigt der Papierwert der $\gg$ Finanzströme《 bei weitem den des Welthandels und ist noch im Wachsen begriffen. Doch von solchen Summen kann nicht einfach auf Macht geschlossen werden. Gehandelt werden Eigentumstitel auf Rohstoffe, Güter und (in zunehmendem Maße) Dienstleistungen, die allesamt stärker lokal fixiert sind und daher vermutlich auch ein gewisses $\mathrm{Ma} ß$ an nationaler Identität besitzen.

Zweitens ist nicht klar, wie effektiv die makroökonomische Planung im Nordwesten jemals war. Sie schien effektiv zu sein, als es noch massives Wachstum gab und die Regierungen Zugriff auf die Überschüsse hatten. Viele konnten gemäßigt interventionistisch agieren (obwohl selektive Anreize im allgemeinen effektiver waren als physische Kontrollen). Doch seither ist nicht nur der Keynesianismus zusammengebrochen, sondern die ökonomische Theorie überhaupt. Die Ökonomen geben heute mehr oder weniger offen $z u$, daß sie keine Erklärung auch nur für einen der großen Booms oder für die Depressionen des 20. Jahrhunderts haben (oder zumindest keine, die nicht auf singuläre Ereignisse wie die beiden Weltkriege zurückgreift). Die makroökonomische Planung war eine allgemeine Ideologie, die von hochabstrakten Konzepten umgeben war, aus denen mit zweifelhaften Begründungen einige technische Verfahren (vor allem die volkswirtschaftliche Gesamtrechung) und wirtschaftspolitische Strategien abgeleitet wurden (die ebenfalls wiederum auf Kontingenzen beruhten). Die makroökonomische Planung beruht immer noch auf dieser Mixtur, auch wenn die Emphase geschwunden ist. Die ideologischen Prätentionen wurden ebenso zurückgeschnitten wie die Möglichkeiten zur Ausgabensteigerung. Von daher sind laxere und fiskalisch vorsichtigere nationale und internationale (z.B. trilaterale) makroökonomische Strategien zu erwarten: mannigfache Richtlinien der G 7 und von GATT sowie schrittweise Liberalisierungsprogramme; eher Zusammenarbeit und Anreize à la MITI und 
weniger Nationalisierung oder direkte staatliche Investitionen; eher Zentralbank- als Regierungspolitik; weniger Ansprüche, den Markt zu kontrollieren, als Signalisierung von Absichten; und vor allem keine Steuererhöhungen, die als großangelegtes ökonomisches Konzept maskiert sind.

Auch die Gründe für die nicht unbedingt dramatischen Machteinschnitte sind nicht leicht zu deuten. Während sich die Wirtschaft internationalisiert hat, stagnieren weltweit die realen Lebensstandards und Ungleichheiten (mit Ausnahme von Ostasien). Wenn den nationalen Regierungen in ihrer ökonomischen Planung und den wohlfahrtsstaatlichen Ansprüchen zusehends Grenzen gesetzt sind, so kann man dies entweder auf transnationale Entwicklungstrends oder auf Rezessionen zurückführen; Transformationen wie die $\gg$ Restrukturierung « mögen eine Antwort auf beides sein. Die $\gg$ Importsubstitution « in Lateinamerika zum Beispiel gedieh aufgrund der regionalen wirtschaftlichen Expansion, die durch den Zweiten Weltkrieg ermöglicht wurde und sie brach unter der Schuldenlast zusammen, die sich wegen des billigen Geldes in den siebziger Jahren angehäuft hatte. In den achtziger Jahre folgten dann Stagnation und Inflation. Die »)Restrukturierung überzieht die Region gegenwärtig in extremem Ausmaß, wischt die nationale makroökonomische Planung praktisch beiseite und stutzt den Wohlfahrsstaat zusammen. Doch mag dies weniger am Transnationalismus liegen als an der Macht, die dem Finanzkapital durch die Schuldenaufnahme überlassen wurde: Die Gläubiger können die Rückzahlungsbedingungen festsetzen. Dabei handelt es sich um die übliche gemischte Gruppe aus Banken mit nationaler Identität und transnationalem Geschäftsfeld sowie internationalen und vorwiegend nördlichen Institutionen wie Weltbank und IWF. Hinzu kommt die amerikanische Regierung, die einerseits die amerikanischen Investoren schützen, andererseits die Region geopolitisch und geoökonomisch freundlicher stimmen bzw. unterwerfen will. Im Gegensatz dazu kann jedoch die heutige »Restrukturierung« in Korea eine bloße Umorientierung der stärkeren makroökonomischen Politik sein, weil das Land dank des ökonomischen Wachstums seine beträchtlichen Schulden zurückzahlen und weitere ausländische Investitionen anziehen konnte.

Auch die Fiskalkrise der meisten Staaten des Südens und Nordens könnte eher der Rezession als einem transnationalen Kapitalismus geschuldet sein. Aufgrund meiner früheren Arbeiten (Mann 1986, 1993a) kann ich mit einiger Sicherheit sagen, daß zumindest seit dem 13. Jahrhundert die Bürger nur in Kriegszeiten einigermaßen bereit waren, höhere Steuern zu bezahlen. Ihre Widerspenstigkeit während der friedlichen siebziger Jahre und später, in einer Rezessionsphase (als die Realeinkommen stagnierten oder fielen), überrascht kaum. Das ist der historische Normalfall, nicht die besondere Auswirkung der »Postmoderne $\ll$ oder des »Globalismus«. Politische Bewegungen, 
die sich traditionell auf den Nationalstaat verlassen, wie die Sozial- und Christdemokratie und die amerikanische Demokratische Partei sind in der Tat in eine Art Krise geraten. Ihre Entwicklung ist gestoppt und leicht rückläufig (eher was ihre Fähigkeit angeht, radikale politische Konzepte zu entwerfen und umzusetzen, als was ihre Attraktivität für die Wähler betrifft). Wiederum ist nicht ganz klar warum. Sind die neuen Mächte des transnationalen Kapitals dafür verantwortlich (und in Europa vielleicht auch die europäischen Institutionen) oder die Bürger, die sich weigern, bei stagnierenden oder schrumpfenden Realeinkommen eine Politik hoher Steuern und Ausgaben zu tragen? Vermutlich beides, doch bisher kenne ich keine Forschungsarbeit, die klar zwischen diesen beiden rivalisierenden Hypothesen unterscheiden könnte. Sollte natürlich das Wirtschaftswachstum so gering bleiben oder durch seine Ungleichmäßigkeit die gesellschaftliche Ungleichheit und die Arbeitslosigkeit verschärfen, ähnelten die Effekte der politischen Schwächung der linken Mitte vermutlich jenen, die von den Enthusiasten ausgemacht wurden. Das sozial orientierte Staatsbürgerschaftsregime im Norden hat offenbar seine Blüte hinter sich und ist leicht schwindsüchtig. Doch könnte dies durch eine Reihe künftiger Entwicklungen umgekehrt werden: wirtschaftliche Erholung, veränderte demographische Trends (sollte z.B. durch eine alternde oder besser ausgebildete Bevölkerung die Arbeitslosigkeit und damit die Ungleichheit reduziert werden) oder politische Kehrtwendungen.

Doch auch die nationalen Ökonomien unterscheiden sich beträchtlich - hinsichtlich Reichtum, Kohäsion und Macht. Betrachten wir zunächst die drei Hauptregionen des Nordens. Nordamerika wird von seiner Supermacht USA beherrscht. Sie hat einen ungewöhnlichen Staat, der von einer einzigartigen Kriegsmaschine und einem (recht mageren) sozialen Sicherungssystem beherrscht wird. Die meisten anderen Regierungsaktivitäten, die in anderen Ländern des Nordens hauptsächlich zur Domäne des Zentralstaats gehören (Strafjustiz, Bildung und Erziehung, Wohlfahrtsprogramme), sind Angelegenheit der 50 Bundesstaaten der USA. Drei wichtige Industriezweige, die Landwirtschaft, der militärisch-industrielle Komplex und das Gesundheitswesen, sind eng mit der Bundesregierung verflochten und können als ansatzweise (wenn auch partikularistisch) geplant bezeichnet werden. Dies wird vermutlich auch so bleiben - obwohl es gegenwärtig Vorhaben gibt, das Militär innerhalb von 20 Jahren um etwa ein Viertel abzubauen. Viele andere Industrien haben recht enge Beziehungen zu bundesstaatlichen Regierungen und lokalen Behörden, etwa die Immobilien- und die Bauwirtschaft. Die Gesetzgebung des Bundes ist auf dem Feld der industriellen Beziehungen und der Monopole traditionell restriktiv - und beschränkt vor allem die Entwicklung von Gewerkschaften und das Wachstum der Banken. Doch auf keiner politischen Ebene gibt es eine nennenswerte makroökonomische Planung. 
Die eigentliche $»$ Planungs «-Behörde ist die Federal Reserve Bank (mittels der Zinssätze), die gegenüber der Regierung weitgehend autonom ist. Es gibt keine ernsthafte amerikanische Industriepolitik, sie wird den nach dem Krieg entstandenen Kraftwerken der amerikanischen Wirtschaft überlassen, den großen Unternehmen. Dies geht vor allem auf die radikale, in der Verfassung festgeschriebene Gewaltenteilung zurück. Eine politische Ökonomie kann nicht auf kohärente Weise vom Präsidenten und seinem Kabinett, den beiden Häusern des Kongresses, dem Obersten Gerichtshof und den 50 Bundesstaaten gelenkt werden (die ebenfalls dieser Gewaltenteilung unterliegen) besonders, wenn sie auch noch verschiedenen Parteien angehören. Somit ist nicht klar auszumachen, inwiefern von einer Schwächung der amerikanischen Regierung die Rede sein könnte, da deren Machtbefugnisse nie besonders aktiv ausgeübt wurden. Natürlich bedeutet allein schon die Rezession, daß dies jetzt ohnehin nicht möglich ist. Angesichts des stagnierenden Lebensstandards der Familien kann keine Regierungsbehörde Steuern erhöhen, um Geld hinter irgendeiner Politik herzuwerfen. Hingegen ließe sich unter gewissen anderen Gesichtspunkten sagen, daß der amerikanische Nationalstaat seinen Zugriff festigt. So unterschiedliche Organisationen wie Banken, TV-Sender und Zeitungen werden national stärker integriert, und die Aufnahme von Immigranten in verblüffender Zahl (die Einwanderung erreicht inzwischen wieder das Niveau von vor 1914) durch das Schulsystem und den Arbeitsmarkt läßt auf eine formidable nationale Solidarität schließen.

Natürlich stehen die USA unter dem Einfluß von Veränderungen des Kapitalismus. Der Konkurrenzdruck der beiden anderen Geo-Ökonomien des Nordens wurde vor allem deutlich durch die Einführung des NAFTA, einer Freihandelszone zwischen den USA, Kanada und Mexiko, die möglicherweise einmal auf andere stabile Ökonomien in Zentral- und Südamerika ausgedehnt wird. Obwohl die Ökonomien Kanadas und der USA ähnlich entwickelt und schon partiell integriert waren, hat die Verbindung des »südlichen« Mexiko mit der »nördlichen« USA einige Beobachter dazu veranlaßt, NAFTA als einen Mikrokosmos der neuen globalen Ökonomie zu betrachten. Doch Mexiko exemplifiziert jene »Prinzipien regulärer Ausdehnung«, die ich oben benannt habe. Es ist ein Nachbar, ein Freund und ein sehr stabiler Staat: seit siebzig Jahren von einer Einheitspartei beherrscht, die milden Zwang ausübt, aber bisher in der Lage ist, institutionell auf Druck zu reagieren. Es bietet eine recht gute Infrastruktur und einigermaßen alphabetisierte und gesunde Arbeitskräfte, und die Nation ist von keinem umfassenden Konflikt unter den Bürgern ergriffen. ${ }^{2}$

2 Chiapas ist die einzige Provinz, in der ethnische Konflikte zu erwarten sind, da die Mestizen sonst überall vorherrschen. Der Grund dafür ist, daß Chiapas in den zwanziger Jahren von Guatemala erworben wurde. 
Die amerikanische Hegemonie auf dem Kontinent macht die NAFTA ebenfalls einzigartig - und ganz anders als die Europäische Union. Kanada ist eine fortgeschrittene, aber kleine abhängige Ökonomie mit einem schwachen Staat - der sich vielleicht bald auflöst. Mexiko ist viel ärmer, trägt in letzter Zeit immer schwerer unter einer Schuldenlast und hat etwas von seiner politischen Stabilität verloren. Doch die USA selbst sind am Schwanken, voller Zweifel über den Freihandel und die Einwanderung der Latinos, und ihre politische Zersplitterung macht eine koordinierte Entscheidungsfindung schwierig. NAFTA umfaßt also drei verschiedene Machtprozesse: es handelt sich tatsächlich um eine »Weltökonomie im Kleinen «; doch die Zone wird auch von einem Nationalstaat beherrscht; und diesen kennzeichnet eine besonders fragmentierte Politik und politische Ökonomie. Die Kombination dieser drei Faktoren ist einzigartig auf der Welt, doch wie wir sehen, ist die Verknüpfung transnationaler kapitalistischer Transformationsprozesse mit politischen und geopolitischen Institutionen durchaus normal.

Ostasien wird gegenwärtig ebenfalls von einem Nationalstaat beherrscht, auch wenn Japan keine militärische Supermacht ist. Die politische Ökonomie Japans unterscheidet sich von der nordamerikanischen wie auch von der europäischen. Es gibt dort eine stärkere Koordination zwischen Staat und kapitalistischen Unternehmen (und, in einer abhängigeren Rolle, den Gewerkschaften). Wade (1990) nennt dies »den Markt regieren«, Weiss und Hobson (1995) sprechen von »regierter Interdependenz«. Diese nationale Koordination wurde von den kleinen Ökonomien Ostasiens in dieser oder jener Form übernommen. Dazu gehört etwa eine aktive Industriepolitik mittels selektiver Steuersätze oder bedingter Subventionen für Schlüssel- und Exportsektoren, die öffentliche Abfederung von Innovationsrisiken und die staatliche Koordination der Unternehmenszusammenarbeit bei der Einführung neuer Techniken (Weiss 1995). Diese Länder sind außerdem politisch stabil und haben eine fortgeschrittene zivile, d.h. "nationale« Gesellschaft, die stabil, alphabetisiert und im wesentlichen ehrlich ist. Dort kommt es auch zu phänomenalen Wachstumsraten. In Japan mag das ins Stottern geraten sein, für das restliche Ostasien gilt dies jedenfalls nicht.

Die ostasiatischen Regierungen haben daher eine üppige Steuerbasis und ein Wachstum, das auch Schulden ermöglicht; ihre Länder sind für ausländische Investoren attraktiv. Sie können die Steuern erhöhen, um das Wohlfahrtssystem auszubauen und von einer Position der Stärke aus mit ausländischen Unternehmen verhandeln. Bei ziemlich ausgeglichener Einkommensverteilung gibt es recht großzügige öffentlichen Dienstleistungen wie Ausbildung und Wohnungsbau. Die einheimischen Industrien werden geschützt, wenn auch auf unterschiedliche Weise. Korea und Malaysia hegen ihre eigenen Automobilindustrien in protektionierten Märkten. Thailand 
schlägt einen anderen ostasiatischen Weg ein. Dort sitzen schon japanische Autohersteller, und die großen amerikanischen Unternehmen verhandeln derzeit wegen des Baus von Fabriken im Land. Die thailändische Regierung scheint von einer Position der Stärke aus zu verhandeln. Sie offeriert keine Steuernachlässe und verlangt beträchtliche Anteile für inländische Zulieferer. Die Philippinen bieten einen viel größeren einheimischen Markt, enorme Steuerkonzessionen und stellen keine weiteren Bedingungen. Doch die Autohersteller ziehen Thailand vor. Warum? Ihnen zufolge ist die thailändische Regierung vertrauenswürdiger und stabiler. Die amerikanischen und japanischen Buchhalter können die künftigen Profite und Verluste dort viel präziser berechnen (USA Today, 5. März 1996). Vermutlich machen die Merkmale der Regierung nicht den ganzen gewünschten Unterschied aus. Die thailändische Gesellschaft besitzt wahrscheinlich auch einen höheren Alphabetisierungsstand, mehr Disziplin und Aufrichtigkeit. Doch dies sind allesamt Eigenschaften eines nationalen Netzwerks, des Nationalstaats. In Ostasien gehen kapitalistische Transformationen und Nationalstaaten unterschiedliche Kombinationen ein.

Europa ist die einzige der drei Regionen, in der es zu bedeutenden politischen Transformationen kommt. ${ }^{3}$ Diese schränken die von uns so genannte »partikularistische« Autonomie der Mitgliedsstaaten ein. Sie können in vielen Bereichen keine eigene Politik mehr betreiben - von den Produktauszeichnungen bis zur Folter mutmaßlicher Terroristen. Auf lange Sicht kann sich dies auch auf wichtige konstitutionelle Unterschiede auswirken. Der wachsende Druck der Lobbyisten auf die europäische und die nationalen Regierungen (die heute effektiver und mehr Interessen repräsentieren müssen als in der Vergangenheit), wird vermutlich im Zusammenwirken mit der Regionalpolitik der EU (die viele Geldmittel anbietet) zu einer gleichmäßigeren Verteilung der Macht zwischen Zentral- und Lokalregierungen führen. Auch die verfassungsmäßigen Rechte von Bürgern und Minderheiten nähern sich einander an. Die Staaten konvergieren und verlieren Machtbefugnisse an Brüssel.

Der ursprüngliche Impetus dieser Entwicklung war weitgehend geopolitischer und militärischer Natur: Es ging darum, einen dritten verheerenden Krieg auf dem Kontinent zu verhindern und insbesondere Deutschland in ein friedliches Konzert der Nationalstaaten einzubinden. Die USA hatten ihre eigenen, vorwiegend geopolitischen Gründe, diesen Prozeß gutzuheißen. So wurden die »Sechs « und die »Neun« zusammengeschlossen, noch bevor man von einer Transformation des Kapitalismus sprechen konnte. Da jedoch die bevorzugten Bindemittel vorrangig ökonomischer Natur waren,

3 Ich habe diesen Punkt eingehender und unter Vergleich mit anderen Regionen in einem anderen Artikel erörtert (Mann 1993b). Die in diesem Papier vorgetragen Ansichten zeigen jedoch, daß ich meine Auffassungen seither in vieler Hinsicht geändert habe. 
verstärkte sich deren Wirkung durch diese Transformation. Die Wirtschaft Europas hat sich daher tiefgreifend transnationalisiert.

Jedoch bleibt auch die Europäische Union eine Assoziation von Nationalstaaten, ein internationales Interaktionsnetzwerk. Der Motor seines Wachstums waren immer spezifische geopolitische Vereinbarungen zwischen Deutschland und Frankreich, die von ihren Klienten, den Beneluxstaaten, unterstützt wurden. Deutschland und Frankreich haben wie die anderen Staaten viele partikularistische Autonomien verloren. Doch wenn sie sich zusammenschließen, bleiben sie in den meisten großen Fragen Herr ihrer selbst. Fragt man Deutsche, welche ökonomische Souveränität, und Franzosen, welche politische Souveränität sie verloren haben, fällt ihnen die Antwort schwer. Die kleineren und wirtschaftlich schwächeren Staaten mögen scheinbar mehr verloren haben, doch ihre Souveränität in den großen Fragen war auch in der Vergangenheit recht beschränkt. Großbritannien hat, wegen seiner historischen geopolitischen Unabhängigkeit vom Rest Europas, das meiste verloren. Und sie wählen und übernehmen Ministerien anhand einer Kombination ihrer Bevölkerungsgröße und ökonomischen Stärke. »Sie« sind Staaten und Nationalökonomien, die von Staatsmännern (und -frauen), von nationalen Technokraten und Wirtschaftsleuten repräsentiert werden. Es handelt sich hier nicht um »traditionelle« Geopolitik, da die Agenda primär ökonomisch geprägt ist und die Teilnehmer glauben, daß Krieg zwischen ihnen unmöglich sei. Es geht um »sanfte« Geopolitik, die von viel dichteren internationalen (und den verbleibenden nationalen) Interaktionsnetzwerken strukturiert wird.

Europa hat sich also politisch und ökonomisch verwandelt, wobei es zu einer beträchtlichen Einschränkung der partikularistischen Autonomie und Souveränität der Nationalstaaten kam. Obwohl die Vermittlungsmechanismen dieser Machttransfers weitgehend geopolitischer Natur waren (und vom Enthusiasmus der europäischen Föderalisten getragen wurden), sind sie über Regeln, Institutionen und Praktiken institutionalisiert, die weitgehend transnational werden und die sozialen Erwartungen in ganz Europa verändern. Doch all dies koexistiert mit einer viel dichteren, wenn auch »weicheren « Reihe internationaler Netzwerke. Da die Dichte beider historisch beispiellos ist - weder die delische Liga noch die Hanse oder ein anderes Gebilde haben je so transnational oder international das soziale Leben durchdrungen - haben wir auch keinen politischen Begriff, um dies zu beschreiben. Das politische Vermächtnis der griechischen Sprache ist endlich überholt! Vielleicht ist der beste Ausdruck einfach »Europa«. Wenn ja, dann wird es wohl nicht die Zukunft der Welt sein.

Es wäre unklug, sich generell über »den Süden« auszulassen, da er zu heterogen ist. Ein Teil könnte durchaus Ostasien auf seinem Weg in den Norden 
folgen. Eine echte transnationale Durchdringung würde hier, dort und überall bestimmte Territorien in einem nicht vorherbestimmten Weg integrieren, fast unabhängig von staatlichen Grenzen. Dies ist jedoch eher unwahrscheinlich, da es immer noch an stabilen Regierungen, gesellschaftlicher Ordnung, Bildung und Gesundheit mangelt, um ausländische Investitionen anzuziehen und wirtschaftliche Entwicklung zu ermöglichen, und die geopolitischen Allianzen eine gewisse, wenn auch geringere Relevanz bewahren (falls nicht neue globale Spannungen an die Stelle des Kalten Krieges treten). All dies erfordert gesellschaftliche Organisation, die auf nationaler oder staatlicher Ebene hergestellt werden muß - welche andere Instanz könnte dies leisten? Sollte Chile es in den Norden schaffen, dann deshalb, weil sein ohnehin schon ziemlich stabiler Staat und seine Zivilgesellschaft durch eine entschieden antilinke Politik gestärkt wurden, durch eine staatlich auferlegte Ordnung und einen ebensolchen ökonomischen Neoliberalismus, die für ausländische Investoren, besonders US-amerikanische, attraktiv waren. Sollten die reicheren arabischen Länder es schaffen, dann wegen des Öls - doch dies geht ebenfalls einher mit formidablen Staaten. China und Indien - ein Drittel der Weltbevölkerung - bieten unterschiedliche Kombinationen von massiven ökonomischen Ressourcen, starken Zivil- (d.h. »National «-) Gesellschaften und ambitionierter staatlicher Regulation. Wird die Vorstellung von einer nationalen Staatlichkeit in diesen Ländern durch ökonomischen Erfolg verstärkt oder geschwächt werden? Gewiß wird sie sich verstärken.

Am anderen Ende scheinen sich die tief in Schwierigkeiten steckenden afrikanischen Staaten aus vormodernen und nicht aus modernen Motiven heraus zu fragmentieren. Ihr Anspruch, an der Moderne teilzuhaben, wozu auch die Konstitution von Nationalstaaten gehörte, stand nur auf dem $\mathrm{Pa}$ pier. Der internationale Kapitalismus würde sie gerne aufpäppeln, nicht zersplittern. Doch dazu besitzt er zu wenig Macht auf lokaler Ebene oder eine zu geringe Spanne an Aufmerksamkeit. Es gibt attraktivere Gebiete, mit stärkeren Staaten und Zivilgesellschaften.

Die entscheidende Frage für die Nationalstaaten in weiten Teile der Welt ist gegenwärtig also das Entwicklungsniveau - der Wirtschaft im engeren Sinne, aber auch von zwei Voraussetzungen der wirtschaftlichen Entwicklung: »Zivilität« des Landes und infrastrukturelle Kapazität des Staates. Die zusammenhängende Entwicklung dieser drei Elemente führte im Nordwesten zum Nationalstaat und zu dessen Expansion auf den gesamten Norden. Wenn die Weltentwicklung stagniert, wird auch die Expansion des Nationalstaats stagnieren - manche »papierene « Nationalstaaten könnten sogar zusammenbrechen. Doch wenn Entwicklung möglich ist, wird sie in jenen Ländern geschehen, die am meisten den Nationalstaaten ähneln, denen sie wiederum zur Expansion verhelfen wird. 
Doch angenommen, die Ökonomie treibt immer mehr in Richtung transnationaler Globalismus, das Ziel des freien Welthandels wird weitgehend erreicht, wenn sich EU, NAFTA, die asiatischen und pazifischen Konferenzländer und andere Handelsbündnisse unter dem Dach des GATT vereinen, angenommen, daß die Multis immer kosmopolitischer werden, daß die Entwicklung des Südens diffundiert und sich weniger um den Nationalstaat zentriert? Würde dies auf eine einzige transnationale/globale Ökonomie hinauslaufen, in der die Ware und der eine Markt die universelle Herrschaft ausübten?

Die Antwort lautet ja und nein zugleich. Alle Güter und Dienstleistungen hätten dann einen Preis auf dem einen Markt, und die kapitalistischen Unternehmen würden ihre Finanzierung, Produktion und ihren Austausch organisieren. Der »Konsumismus« hat schon die Oberhand ergriffen, sagen manche Enthusiasten; privatwirtschaftliche Bilanzierungsverfahren verbreiten sich auch in zuvor abgeschotteten Institutionen wie den öffentlichen Diensten und Universitäten. Und die Sportler verkaufen ihre Fähigkeiten an den höchsten Bieter auf freien und relativ jungen Märkten. Diese Art von Kommodifizierung würde sich verstärken.

Aber selbst dann hätten die Regeln dieser Märkte noch ihre partikularen Züge, manche davon die Wirkung nationaler und internationaler Interaktionsnetzwerke. Obwohl heute eine weit größere Spanne an Gütern gekauft und verkauft werden, werden viele der wichtigsten nicht wirklich als Waren auf freien Märkten gehandelt. Keine der drei größten Industrien der USWirtschaft, Verteidigung, Gesundheitswesen und verbotene Drogen, unterliegen ohne weiteres den Regeln der Warenproduktion, obwohl bei allen beträchtliche transnationale Netzwerke im Spiel sind. In der Verteidigungsindustrie ist die Regierung ein monopolistischer Nachfrager für hochtechnisierte Waffensysteme, und sie entscheidet auch, welche anderen (befreundeten) Staaten als weitere Nachfrager zugelassen werden. Das Angebot ist nicht besonders konkurrenzbestimmt (gelegentlich macht nur ein einziger Hersteller »ein Angebot«, oder der Profit wird einfach auf die Kosten aufgeschlagen). Die Waffen verkörpern mehr »Gebrauchs《- als »Tausch«-Wert, die USA muß sie haben und das Unternehmen kann sie produzieren, ohne sich Marktrisiken zu sorgen. Die Gesundheitsindustrie offeriert ihre Waren unter stärkerem Konkurrenzdruck, sie besitzt jedoch eine eigentümliche mehrschichtige Struktur mit enormem bürokratischen Aufwand (der Versicherungsunternehmen, Krankenhäuser etc.), die wiederum nach Zahlungsfähigkeit der Kunden organisatorisch differenziert ist. Und auch hier gilt, daß für den zahlungsfähigen Kunden das Produkt eher ein Gebrauchs- denn ein Tauschwert ist. Die Bewahrung der Gesundheit (definiert gemäß den gängigen medizinischen Verfahren und Machtverhält- 
nissen) wird fast um jeden Preis angestrebt. Natürlich sind bei beiden Industrien große multinationale Unternehmen im Spiel, und die globalen Finanznetzwerke sind mit Investitionen dabei. Doch diese fließen in Organisationen mit ausgeprägt nationalem und (im Falle der Verteidigungsindustrie) internationalem Charakter. In der dritten Industrie, dem Geschäft mit den verbotenen Drogen, scheint die Belieferung der Kunden weitgehend Tauschcharakter zu haben. Doch die Industrie wird auch geprägt durch ihre Schnittstellen mit Justiz und Polizei sowie mit eindeutig kriminellen, geheimen und gewalttätigen Organisationen. Die Sucht produziert auch Konsumenten, für die das Produkt ein Gebrauchswert ist, der um fast jeden Preis (auch Verbrechen) erworben werden muß. Die Ware muß also nicht unbedingt herrschen, auch nicht in einer als kapitalistisch dominiert erscheinenden Wirtschaft. Die Ökonomie enthält diverse gesellschaftliche Praktiken und Werte, die der Herrschaft des Warentauschs eigene »Blockaden « entgegenstellen.

Obwohl die kapitalistische Ökonomie heute wesentlich »globalen« Charakter hat, ist dieser Globalismus »nicht rein«, nämlich eine Kombination des Transnationalen und Internationalen. Der potentielle Universalismus des letzteren wird durch die Partikularismen der Nationalstaaten untergraben - und auch durch die Partikularismen gesellschaftlicher Praktiken insgesamt.

\section{Umweltgefährdungen, neue sozialen Bewegungen und eine neue transnationale Zivilgesellschaft}

Bevölkerungswachstum, Boden- und Pflanzenerosion, Wasserverknappung, Verschmutzung der Atmosphäre und Klimaveränderungen stellen für uns eine zweite Form des Globalismus dar - noch verstärkt durch die Gefahren eines biologischen, chemischen oder nuklearen Krieges, auf die wir später eingehen. Wir leben tatsächlich, und erst seit Mitte des 20. Jahrhunderts, in der Beck'schen »Risikogesellschaft « (doch ist dies nicht die einzige, in der wir leben). Bei einigen dieser Probleme funktioniert auch weiterhin die traditionelle $»$ Lösung «, den Süden oder die Armen hungern zu lassen. Doch andere stellen schwere Risiken für die Menschheit insgesamt dar. Sie sind nicht identisch mit denen des Kapitalismus, wenn auch eng mit diesen verflochten (da der Kapitalismus nicht die dominante Form der ökonomischen Produktion ist). Die »Unterwerfung« und »Ausbeutung « der Natur und die enorme Zunahme der entsprechenden Möglichkeiten sind ebenfalls dem Industrialismus und anderen in der Moderne entwickelten Produktionsformen zuzuschreiben. Der Staatssozialismus (und auch der Faschismus) war sogar noch zerstörerischer gegenüber der Umwelt, während der einfachen Warenproduktion von Kleinbauern ebenfalls viele destruktive Praktiken auf- 
gezwungen wurden. Nationalstaaten, wissenschaftliche Establishments und (bis in die letzen Jahre) praktisch alle modernen Institutionen haben ihren Beitrag zur Zerstörung geleistet. Und das rapide Bevölkerungswachstum hat ebenfalls Ursachen, die außerhalb des Kapitalismus liegen - etwa militärische, religiöse und patriarchalische Praktiken. Antworten auf diese Risiken müssen über den Nationalstaat und den Kapitalismus hinausgehen.

Die gegenwärtigen Reaktionen auf Umweltprobleme lassen sich grob zweiteilen. Erstens gibt es jetzt schon Organisationen, welche die bekannte Maxime »Global denken, lokal handeln « in vielerlei Gestalt umzusetzen versuchen. Es handelt sich vor allem um gemischte lokal-transnationale Pressure-Gruppen und NGOs, manche formell organisiert (wie Greenpeace), andere von Netzwerken aus Spezialisten und Wissenschaftlern getragen. Sie sind eher »modern« als »postmodern«, da sie die wissenschaftlich-materielle Ausbeutung der Natur aus vorwiegend natur- und sozialwissenschaftlichen Gründen ablehnen. Obwohl diese Eliten zunächst im Norden entstanden sind, verbreiten sie sich zunehmend auf dem Globus, unter den hochgebildeten Eliten des Südens und unter diversen, eher partikularen Gruppen, die von realen materiellen Problemen bedroht sind. Solche Netzwerke setzen die modernsten und globalsten Kommunikationsmittel ein. Indem sie diese nutzen, umgehen sie manchmal die nationalen Regierungen und das internationale Kapital - so etwa 1995 bei der Mobilisierung von Konsumenten in Westeuropa für den Shell-Boykott, als die britische Regierung gedemütigt wurde und die Ölbohrplattform Brent Spar wiedervertäut werden mußte. Davon steht noch mehr zu erwarten.

Handelt es sich hier um eine »globale Zivilgesellschaft $\ll$ ? Die Struktur ist nicht ganz neu: Anfang des Jahrhunderts schufen die Sozialisten (und, in geringerem Maße, auch Anarchisten, Pazifisten und Faschisten) ausgedehnte transnationale Netzwerke, die sich über weite Teile des Globus erstreckten und ebenfalls mit avancierten technischen Mitteln arbeiteten (Druckerpressen, Sofortübersetzung, Diktaphone - man denke etwa an Trotzkis bemerkenswerte Studie in Mexico City). Die Sozialisten brachten eine Welle von Revolutionen in Gang, manche erfolgreich, die meisten erfolglos. Viele der idealistischeren Verfechter einer neuen Zivilgesellschaft erwarten, daß sie ihre historischen Vorläufer dereinst klein aussehen läßt.

Zweitens jedoch werden auch in wachsendem Maße interstaatliche Gremien und Institutionen gegründet, makroregionale und kontinentale Gebilde, UN-Konferenzen usw. Deren wichtigste Teilnehmer sind Repräsentanten von Nationalstaaten, die koordinierte Politikentscheidungen implementieren könnten. Auch eine »sanfte Geopolitik « wird in diesen Arenen verstärkt propagiert. Andere wichtige Delegierte sind die oben erwähnten »Experten«, die ein Doppelleben führen. Obwohl in transnationalen Berufsverbänden 
großgeworden, müssen sie die Perspektive des Nationalstaats übernehmen und die Regierungen davon überzeugen, daß globale Sichtweisen tatsächlich im nationalen Interesse sind. Manchen gelingen dabei tolle Streiche. Amerikanische Ornithologen haben es geschafft, das State Department dazu zu bringen, in sein Hilfsprogramm für Belize die Forderung aufzunehmen, einen seltenen Vogel zu schützen, von dem die Verantwortlichen in Belize noch nie gehört hatten. Bedeutsamer ist schon, daß Feministinnen in Entwicklungsorganisationen reaktionäre Diktatoren des Südens dazu drängen, mehr Mittel in die Ausbildung von Frauen zu stecken, da dies zur Senkung der Geburtenrate führt (eines der Hauptziele fast aller Regierungen des Südens).

Die Behandlung von Umweltproblemen führt meist zu dualen Interaktionsnetzwerken. Auf der einen Seite steht eine potentiell lokale/transnationale Zivilgesellschaft, auf der anderen Seite die internationale „sanfte« Geopolitik. Erstere kann den Nationalstaat transzendieren, letztere die Staaten stärker koordinieren, wenn auch vielleicht in zum Teil konsensuellem Rahmen, der mit der graduellen Verbreitung einer Zivilgesellschaft kompatibel ist. Wiederum eine vielschichtige Angelegenheit.

Dies gilt auch für die anderen »neuen sozialen Bewegungen«. Meist wird argumentiert, daß die mit der »neuen Politik« der Identität befaßten Bewegungen - um Geschlecht, Sexualität, Lebensstile, Alterskohorten, Religion und Ethnizität - die nationalen Identitäten (und die national regulierten Klassen) schwächen und sie durch lokale-plus-transnationale Identitätsquellen ersetzen oder ergänzen. Ethnische Politiken sind zu variabel, um sie in ein paar Absätzen behandeln zu können (ich lasse mich an anderer Stelle ausführlicher dazu aus). Ein Satz soll daher genügen: Ethnische Politik mag die vorhandenen Staaten fragmentieren, doch die Folge dieser Zersplitterung - angesichts der Niederlage von alternativen multinationalen und sozialistischen Staaten - sind noch mehr, angeblich authentischere Nationalstaaten. Was andere auf Identitätspolitik beruhende soziale Bewegungen angeht, so möchte ich behaupten, daß sie die bestehenden Nationalstaaten letztendlich stärken.

Ich habe oben ausgeführt, daß der »moderate Nationalstaat « sich darauf gründete, daß er sich aus dem als »privat« betrachteten Bereichen des sozialen Lebens heraushielt. Besonders heilig war die Familie, und hier mischte sich der Staat nur bei den ganz Armen ein. Säkularen Staaten fehlten im allgemeinen eigene moralische Themen, sie übernahmen die moralischen Konzepte der Religionen. Ihre Gesetze mögen bestimmte Verhaltensformen streng verbieten, doch die Regierungen verließen sich stärker auf die Internalisierung der Moral durch die Bürger als auf die gewaltsame Durchsetzung. Wo die Bürger sich nicht fügen wollten, wichen sie privat aus - und 
den Staaten fehlte es meist an effektiven Infrastrukturen zur gewaltsamen Durchsetzung der Regeln. Offensichtliche Ausnahmen - das Verbot der Kinder- und Frauenarbeit war im 19. Jahrhundert eine wichtiges Gesetzgebungsmaßnahme - gingen auf den Glauben zurück, daß sie der patriarchalen Familie und dem christlichen Sexualverhalten entgegenliefen.

Das 20. Jahrhundert hat dies geändert, durch neue politische Bewegungen und die Durchdringung des Privatbereichs mit dem Wohlfahrtsstaat. Die Staaten sind nun aufgefordert, moralisches Verhalten im bisher als privat betrachteten Bereich gesetzlich zu regeln und durchzusetzen. Ich kann nun nicht mehr durch Rauchen öffentliche Räume verpesten; auch mein Hund kann nicht überall sein Geschäft verrichten. Ich kann nicht mehr meine Frau und meine Kinder schlagen. Wenn ich sie verlasse, muß ich für ihr Wohl sorgen. Viele der neuen Gesetze entsprechen paradoxerweise keinem restriktiven Geist, sondern der Ausweitung persönlicher Freiheiten. Schwule können ihren Lebensstil offen praktizieren; Frauen können eine nichtgewollte Schwangerschaft abbrechen. Doch dies resultiert nicht in einer neoliberalen Abwesenheit staatlicher Regulation - das wäre nur der Fall, wenn man sich letztgültig darauf einigen könnte, was öffentlich und was privat ist. Die Folge ist vielmehr eine unablässige, höchst erregte politische Debatte und ein Strom von Gesetzen. Sollen Schwule heiraten, Kinder aufziehen, zum Militär gehen, Pfadfindergruppen leiten dürfen? Bis zu welchem Monat, aus welchen Gründen, auf welche Weise und in welchen Kliniken dürfen Frauen abtreiben? Hat der mutmaßliche Vater ein Mitspracherecht? Für all diese Fragen und für die komplizierten sozialstaatlichen Ansprüche, die sie nach sich ziehen, brauchen wir gesetzliche Regelungen. Daher werden Pressure Groups gegründet, die dann mit aller Leidenschaft »Kulturkriege « organisieren. Die USA sind ein Extremfall - beide großen politischen Parteien sind zum Teil von diesen »neuen sozialen Bewegungen« okkupiert. Doch auch die meisten Länder der Erde sind heute durch solche moralischen Fragen politisiert.

Diese Kulturkriege beziehen einige transnationale und globale Netzwerke mit ein. Feministinnen, Schwule, religiöse Fundamentalisten usw. nutzen neugebildete globale Kommunikationsnetzwerke und NGOs und konzentrieren ihre Kräfte auf die UNO wie auch auf ihre eigenen Staaten. Allerdings fordern die meisten Akteure in diesem Kampf von ihrem Staat mehr Regulation durch juristische oder sozialstaatliche Instanzen: Restriktion oder Liberalisierung der Abtreibung, des vorehelichen Geschlechtsverkehrs und der Elternschaft von Singles; klare rechtliche Definitionen der sexuellen Belästigung, des Kindesmißbrauch und der Vergewaltigung; die Garantie oder Restriktion der Rechte jener Menschen mit unorthodoxen sexuellen Vorlieben oder Lebensstilen. Da die soziale Regulierung überwiegend im 
Autoritätsbereich der Nationalstaaten bleibt, könnte die Ausbildung neuer Identitäten letzlich zur Wiederbelebung seiner Politik und zur Ausweitung seiner Zuständigkeit führen. Die neuen sozialen Bewegungen behaupten, mit Klassenpolitik wollten sie nichts am Hut haben. Vielleicht ist die Klassenpolitik im Niedergang begriffen - doch nicht die nationale Politik.

\section{Postmilitarismus und die neue Weltordnung}

Martin Shaw zufolge haben die Nationalstaaten des Nordens im Bereich der harten Geopolitik die radikalste Transformation durchgemacht - weil sie hier die bittersten Lektionen gelernt haben. In den beiden Großen Nördlichen Kriegen (bekannt als Weltkriege) hatten sie etwa 70-80 Millionen Tote zu beklagen - als direkte Folge des Systems der Nationalstaaten. ${ }^{4}$ In diesen Kriegen haben sie außerdem verheerende Waffensysteme entwikkelt, die nicht mehr für irgendeine rationale »harte Geopolitik« einzusetzen sind. Die Staaten des Nordens sind heute weniger bereit, sich in ausgewachsene Kriege zu stürzen, als fast jeder Staat der Geschichte. Das ursprüngliche Rückgrat des Nationalstaats verwandelt sich in Gelatine.

Doch wiederum gibt es Unterschiede zwischen den drei Regionen. Militärisch sind die Europäer, die verantwortlichen Protagonisten beider Kriege, zurückhaltender als alle andern. Sie verlassen sich in Sachen Verteidigung auf die USA und sind in ihrer Sicherheit gegenwärtig nicht ernsthaft bedroht. Die EU hat zwar zwei Nuklearmächte, die französisch-deutsche Brigade und die eigentümliche Westeuropäische (Verteidigungs-) Union, doch spielt dies eine viel geringere Rolle als das historisch beispiellose Fehlen einer ernsthaften »harten Geopolitik « in Europa. Die Deutschen bleiben durch den Antimilitarismus am stärksten gebunden. Die Entschlossenheit, mit dem schrecklichen Charakter der europäischen Geschichte zu brechen, ist vermutlich die kausal am stärksten determinierende Transformation der Moderne, die auch die traditionelle nationale Souveränität am stärksten unterminiert. Doch die europäische Geschichte zum allgemeinen Muster für die Welt zu machen, wäre äußerst ethnozentristisch. Und wenn sie es wäre, erforderte die Analogie mehr als bloß die durch eine »kulturelle Wende« verstärkte Restrukturierung des Kapitalismus. Die Analogie würde verlangen, daß in anderen Regionen Millionen in künftigen Kriegen sterben, damit die Leute endlich »Genug! « rufen.

Doch die meisten Japaner haben wohl ebenfalls »Genug! « gerufen. Sie sind gegenwärtig zurückhaltende Militaristen. Manche japanischen Politiker

4 Natürlich hatten diese Kriege komplexe Ursachen. Wie ich jedoch für den ersten Krieg zu zeigen versucht habe (Mann 1993a, Kap. 21), beruhen sie stärker auf den Institutionen des Nationalstaats als auf jeder anderen Organisation von Macht (wie etwa dem Kapitalismus). 
sind kühner als ihre deutschen Kollegen, was nationalistische Töne anbelangt, doch sie kriegen immer noch eins über den Mund. Doch ist Ostasien eine potentiell unsichere Region. Wiederum anders sind die Vereinigten Staaten. Sie haben wenig unter den beiden Großen Nördlichen Kriegen gelitten - im Gegenteil, die Wirtschaft hat stark profitiert. Die USA sind eine militärische Supermacht, die immer noch mit einem stehenden Heer von l 400000 Soldaten ins nächste Jahrhundert gehen will und die Ausrüstung modernisiert. Sie bleiben der Weltpolizist, eine Rolle, in der sie die europäischen und japanischen Regierungen auch weiterhin sehen wollen und die vielleicht sogar der Finanzlage nützt. Doch selbst in den USA gab es beträchtliche Einschnitte in das Verteidigungsbudget, und es ist zweifelhaft, ob die amerikanischen Wähler einen Krieg durchhalten würden, in dem viele ihrer Landsleute getötet werden. Auf jeden Fall beherrschen diese nördlichen Regionen die Welt ohne Krieg.

Die Welt bleibt dennoch von Konflikten durchzogen, in denen die »harte« Geopolitik eine wichtige Rolle spielt. Betrachten wir diese Liste: wachsender ethnischer Separatismus, Konflikte zwischen potentiellen Nuklearstaaten wie Indien und Pakistan oder den beiden Chinas, Chinas geopolitische Rolle entspricht nicht seiner realen Stärke, die Instabilität Rußlands und einiger kleinerer, gutgerüsteter Mächte, die Vorherrschaft von Militärregimen in der Welt, die wahrscheinliche Proliferation von Nuklearwaffen und die weitgehend unkontrollierte Verbreitung von chemischen und biologischen Waffen in der gegenwärtigen Welt. Wer weiß, was für ökologisch bedingte Krisen, etwa aufgrund von Wassermangel, oder was an von außen beherrschter Ausbeutung der Lebensgrundlage eines Landes usw. auf uns zukommt? Es ist unwahrscheinlich, daß Militarismus oder Krieg einfach verschwinden. Alle diese Bedrohungen bilden schwerwiegende Hindernisse für die Diffusion transnationaler und universaler globaler Netzwerke.

Die Gefahren könnten möglicherweise durch eine globale geopolitische Ordnung eingedämmt werden, auch wenn diese zum Teil segmentiert wäre. Sie wird auf absehbare Zukunft um die USA zentriert sein, flankiert vielleicht durch eine verstärkte Koordination mit den größeren Staaten des Nordens und mit den Vereinten Nationen. Shaw zufolge bietet deren Kombination die Grundlage für die Herausbildung einer Weltordnung, er schränkt jedoch ein, daß es sich nicht um einen echten »Staat« handle und daß sie zerrissen bleibe zwischen den »westlichen« und den »globalen «Komponenten. Tatsächlich handelt es sich offenbar um eine Triade, da ihr Kern nicht westlich, sondern amerikanisch ist - womit eine weitere Ebene der Unberechenbarkeit hinzukommt. Die amerikanischen Wähler wollen vielleicht nicht die »Söldner « bereitstellen, die als Weltpolizisten auftreten sollen. Vielleicht reicht es für die Nachbarn, für ein paar strategisch wichti- 
ge Orte und die elementaren amerikanischen Interessen wie Öl, aber nicht für den großen Rest der Welt - oder die mächtigeren unter den Rivalen. Es ist offenbar ein langer Weg zu einer transnationalen oder geopolitischen Ordnung für die Welt insgesamt.

Und selbst die oben erwähnten kriegsähnlicheren Szenarios wären nicht wirklich mit der fürchterlichen Vergangenheit der Nationalstaaten gleichzusetzen. »Harte« Geopolitik - das heißt schreckliche Kriege - war die Ursache für sein anfängliches Wachstum und blieb bis in jüngste Zeit ein wesentlicher Bestandteil des Nationalstaats. Die harte Geopolitik ist heute im Norden vergleichsweise rückläufig, allerdings nicht überall. Obwohl die von den Kriegswaffen ausgehenden Gefahren sich verschärft haben, reduzieren sie im Grunde die Mobilisierungsmacht von Staaten. Wenn Staaten einen Teil ihres traditionellen Kerns verloren haben, sind sie dann im allgemeinen Niedergang begriffen? Das Argument scheint im Hinblick auf Europa am plausibelsten, am wenigsten gilt es für weite Teile des Südens. Außerdem haben wir gesehen, daß die »weiche« Geopolitik Konjunktur haben könnte als Ergänzung der harten Spielart, untermauert von den oben beschriebenen neuen sozialen Bewegungen auf nationaler Ebene.

\section{Folgerungen}

In diesem Aufsatz wurden vier vermeintliche $»$ Gefahren« für den heutigen Nationalstaat analysiert: Transformation des Kapitalismus, Umweltgefahren, Identitätspolitik und Postmilitarismus. Wir müssen auf Abstand gehen von den enthusiastischeren Globalisten und Transnationalisten. Mit wenig historischem Gespür übertreiben sie die einstige Stärke der Nationalstaaten; mit wenig Gespür für die globale Vielfalt übertreiben sie deren gegenwärtigen Niedergang; mit wenig Gespür für deren Pluralität unterschätzen sie die Rolle der internationalen Beziehungen. In allen vier Bereichen der »Gefährdung« müssen wir unterscheiden zwischen (a) unterschiedlichen Auswirkungen verschiedener Staatstypen in verschiedenen Regionen; (b) den Nationalstaat schwächenden und stärkenden Trends; (c) Trends, die nationale Regulationsmechanismen auf internationale und transnationale Netzwerke verlagern; und (d) Trends, die Nationalstaaten und den Transnationalismus zugleich stärken.

Ich habe einige Verallgemeinerungen riskiert. Die kapitalistische Transformation scheint die fortgeschrittensten Nationalstaaten des Nordens in gewissem Ausmaß zu schwächen, doch eine erfolgreiche wirtschaftliche Entwicklung würde sie anderswo stärken. Der Niedergang des Militarismus und der »harten Geopolitik« im Norden schwächt hier den Traditionskern des Nationalstaats. Doch die ersten drei angeblichen »Gefährdungen« sollten die internationalen Netzwerke der »weichen Geopolitik« intensivieren und 
dichter knüpfen. Und die Identitätspolitik könnte (im Gegensatz zur herrschenden Meinung) die Nationalstaaten tatsächlich stärken. Die Phänomene sind zu unterschiedlich und widersprüchlich, die Zukunft zu unabwägbar, als $\mathrm{da} ß$ es uns möglich wäre zu behaupten, der Nationalstaat und das System der Nationalstaaten würden entweder gestärkt oder geschwächt. Vielmehr hat es (entgegen manchen Postmodernisten) den Anschein, daß mit der zunehmenden Integration der Welt die lokalen Netzwerke weiterhin zusammenschrumpfen - obwohl die Fragmentierung mancher heute noch existierender Staaten in kleinere, ethnisch definierte Staaten eine Art Gegentrend darstellen würde, d.h. die Reduktion des Nationalstaats auf eine lokalere Ebene.

Die globalen Netzwerke gewinnen in der Tat an Stärke. Doch sie enthalten drei Elemente. Erstens verdanken sie einen Teil ihrer Stärke dem globaleren Ausmaß der transnationalen Beziehungen, die im wesentlichen den technischen und gesellschaftlichen Beziehungen des Kapitalismus entspringen. Doch haben diese nicht die Macht, den globalen Netzwerken einen singulären Universalismus aufzuzwingen. So sind zweitens auch die globalen Netzwerke zu einem gewissen Grade durch die Partikularitäten der Nationalstaaten segmentiert, besonders der mächtigeren im Norden. Drittens wird diese Segmentierung durch internationale Beziehungen vermittelt. Dazu gehören manche »harten« politischen Strategien, und liefen diese abermals auf Kriege und internationale Spannungen hinaus, würde die Segmentierung tatsächlich zunehmen. Doch gegenwärtig hat die Expansion der »weichen « Geopolitik das größere Gewicht, und diese entspricht eher dem Transnationalismus. Haben wir es mit einer $»$ Weltgesellschaft« zu tun? Nicht im stärksten Sinne, wie die enthusiastischeren Theoretiker oft meinen. Die globalen Netzwerke enthalten keine singuläres, relativ systemisches Prinzip der Interaktion oder Integration. Meine Auffassung von der »Gesellschaft « ist weniger hochgeschraubt, da ich menschliche Gesellschaften immer als multiple, sich überlappende und überkreuzende Interaktionsnetzwerke begreife. Der Globalismus wird dies wohl kaum ändern. Menschliche Interaktionsnetzwerke durchdringen heute die Welt, doch in multipler, variabler und ungleichmäßiger Weise.

Aus dem Amerikanischen übersetzt von Klaus Fritz

\section{Literatur}

Carnoy, M. (1993): Whither the Nation-State? in: Carnoy (1993a).

Carnoy, M. (ed.) (1993a): The New Global Economy in the Information Age, College Park, Penn.: Pennsylvania State University Press.

Castells, M. (1993): The informational economy and the new international division of labor, in: Carnoy (1993a).

Economist (1995): The World Exonomy: Who's in the driving seat? 7. Oktober.

Featherstone, M. (1990): Global culture: an introduction, in: Theory, Culture and Society, Vol. 7. 
Giddens, A. (1990): The Consequences of Modernity, Cambridge: Polity.

Harvey, D. (1989): The Condition of Postmodernity, Oxford: Blackwell.

Lash, S.; Urry, J. (1994): Economies of Sign and Space. London: Sage.

Mann, M. (1986, 1993a): The Sources of Social Power, Vol. 1: From the Beginning to 1760 AD; Vol. 2: The Rise of Classes and Nation-States, 1760-1914, Cambridge: University Press.

-(1993b): Nation-States in Europe and other continents: diversifiying, developing, not dying, in: Daedalus, Vol. 122.

Shaw, M. (1996): The global state: perspectives on the globalization and fragmentation of state power, paper presented to the »Directions of Contemporary Capitalism « Conference, University of Sussex, 26.-28. April 1996.

Taylor, P. (1996): Embedded statism and the social sciences: opening up to new spaces, in: Environment and Planning, im Erscheinen.

Wade, R. (1990): Governing the Market: Economic Theory and the Rise of the Market in East Asian Industrialization, Princeton, NJ: Princeton University Press.

- (1995): Globalisation and its limits: reports of the death of the national economy are greatly exaggerated, in: New Left Review, im Erscheinen.

Weiss, L. (1995): Governed interdependence: rethinking the government-business relationship in East Asia, in: The Pacific Review, Vol. 8.

Weiss, L.; Hobson, J. (1995): States and Economic Development. A Comparative Historical Analysis, Cambridge: Polity Press.

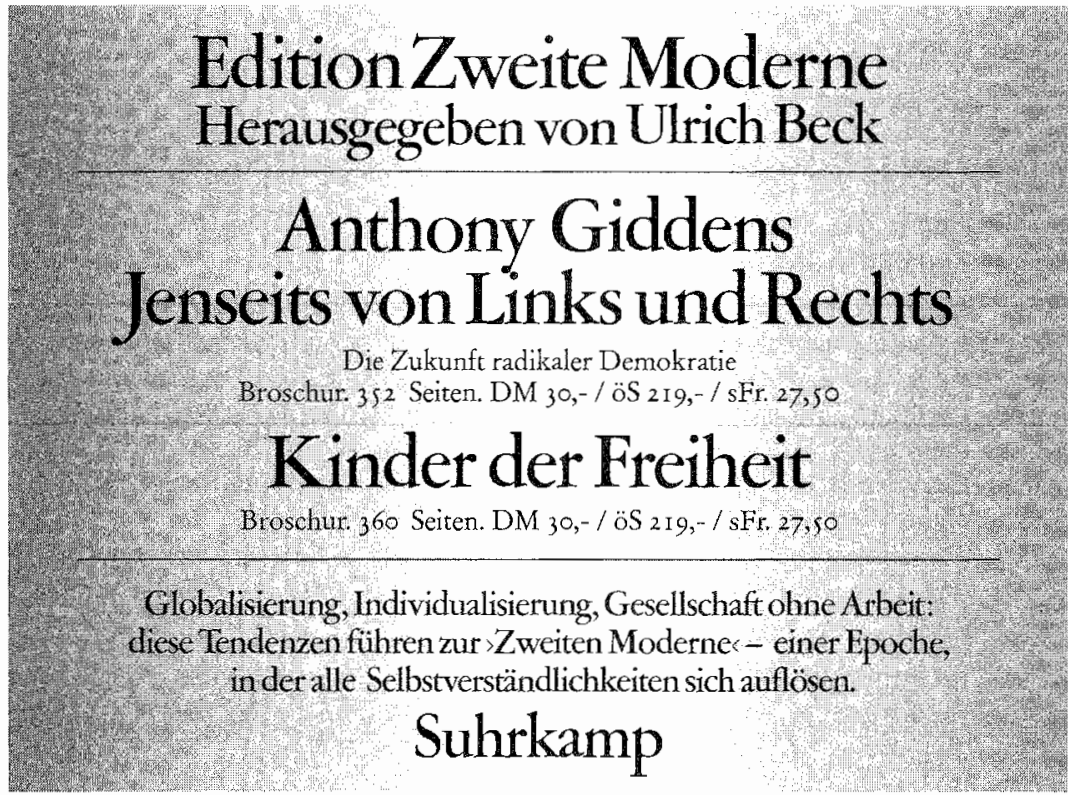

